\title{
Editorial: Expanded Open Access and Creative Commons
}

APS has adopted the guiding principle that its journals will embrace open access publishing to the maximum extent possible, consistent with the financial stability of the Society. In essence, we want to provide our journals to anyone who needs or wants them, as long as we have sufficient income to conduct quality peer review, format and publish articles, maintain and update a large archive, and provide tools for readers to find the ideas and information they need to advance the field.

Accordingly, as of 15 February 2011, authors in most Physical Review journals will have a new alternative: to pay an article-processing charge whereby their accepted manuscripts will be available barrier-free and open access on publication. These manuscripts will be published under the terms of the Creative Commons Attribution 3.0 License (CC-BY), the most permissive of the $\mathrm{CC}$ licenses, granting authors and others the right to copy, distribute, transmit, and adapt the work, provided that proper credit is given. This new alternative is in addition to traditional subscription-funded publication; authors may choose one or the other for their accepted papers.

The new article-processing charges, which will cover all costs and provide a sustainable funding model, have been set at \$1700 for papers in the Physical Review and \$2700 for those in Physical Review Letters. The resulting open access articles will appear alongside and mixed in with subscription-funded articles, converting these journals to 'hybrid' open access journals. Revenue from the article-processing charges will decrease the need for subscription income and help to keep our subscription price-per-article among the lowest of any physics journals.

The most selective of our journals must have higher article-processing charges for their open access articles. Physical Review accepts about $60 \%$ of articles submitted and Physical Review Letters roughly $25 \%$, so the costs are higher than in less selective journals.

In the 15 years since the online journals arrived on the desktops of students and researchers, a series of initiatives have further improved access and diffusion. We have made agreements that bring our journals to previously underserved institutions, researchers, and students. Our increasingly permissive copyright agreement presently allows authors to self-archive the published version of their papers on their own and their institution's websites, to make links to our version from the preprint arXiv, and to create derivative works based on published articles. The new CC-BY license will extend similar rights to others, in addition to the author, as long as credit is given.

The two "Physical Review Special Topics" journals, one in the field of accelerators and beams and the other in physics education research, helped APS gain experience in different funding models for open access, as did the Free to Read program, now replaced by the article-processing fees and Creative Commons license offering. The recent initiative to provide the APS journals online at no charge to on-site users in public libraries and high schools in the United States met the demand for public access to physics research funded by U.S. tax dollars.

In January we announced a new journal, Physical Review X(PRX), an online-only, fully open access, primary research journal covering all of physics and its applications to related fields. As a 'gold' open access journal, PRX opened its doors wide to all fields of physics, including those that have not conveniently fitted within the scope of our existing journals.

Along with diffusing the knowledge of physics, our experiments in open access have confirmed the value to our readers and subscribers of the core publishing services that APS provides: high quality peer review, posting of richly linked articles, and the long-term maintenance of the scholarly record. 
For additional information on the article-processing charges and Creative Commons offerings, please see the journal website.

Gene D. Sprouse

Editor in Chief

American Physical Society

Published 15 February 2011

DOI: 10.1103/PhysRevC.83.020001

PACS number(s): $01.30 . \mathrm{WW}$ 